\title{
La citometría de flujo al servicio del diagnóstico del linfoma en caninos
}

\section{Flow cytometry for the diagnosis of lymphoma in canines}

Rosina Sánchez Solé ${ }^{1}$ 0000-0003-1002-8309

Martín Breijo Dotta ${ }^{2}$ 0000-0002-8871-9428
Cecilia Amaral Oribe ${ }^{3}$ 0000-0002-6835-0395

Paula Pessina Serdio ${ }^{1}$ 0000-0002-7809-9026

${ }^{1}$ Laboratorio de Endocrinología y Metabolismo Animal, Facultad de Veterinaria-UdelaR, Alberto Lasplaces

1620 Montevideo, Uruguay. Email: rosinasanchsole@gmail.com

${ }^{2}$ Unidad de Reactivos y Biomodelos de Experimentación, Facultad de Medicina-UdelaR.

${ }^{3}$ Unidad Oncología, Facultad de Veterinaria-UdelaR

Veterinaria (Montevideo) Volumen 56

№214 (2020 Jul - Dic) e20205621404

\section{Resumen}

El linfoma canino es un tumor maligno que representa del 7 al $24 \%$ del total de neoplasias y el $80 \%$ de los tumores hematopoyéticos. En humanos y en caninos, el $80-85 \%$ de los linfomas se originan a partir de linfocitos B neoplásicos (linfoma B) y un 15 a $20 \%$ son linfomas generados a partir de linfocitos $\mathrm{T}$. Estos últimos en general son más agresivos, presentan menor tiempo de sobrevida y podrían necesitar una terapéutica diferencial. Por tal razón, conocer el inmunofenotipo tumoral (origen del tumor) permite establecer un pronóstico más certero y un tratamiento adecuado al tipo de tumor. En este marco, la inmunofenotipificación por citometría de flujo realizada a partir de una punción de linfonodos es una herramienta de gran valor diagnóstico para la clínica veterinaria. El objetivo del presente trabajo fue presentar los primeros casos clínicos de caninos con diagnóstico clínico y citológico de linfoma, a los cuales se les realizó citometría de flujo para determinar el origen celular de la neoplasia en nuestro país, así como estudios complementarios para determinar repercusiones locales y sistémicas del proceso tumoral. El resultado de la inmunofenotipificación evidenció la presencia de un linfoma de estirpe T CD4+ en una de las pacientes y linfoma B en la otra. A nivel sistémico, la paciente con linfoma $\mathrm{T}$ presentó una leucocitosis neutrofílica e hipercalcemia mientras que en ambas pacientes se observó un aumento de los niveles de la enzima lactato deshidrogenasa. Basados en estos resultados, se discute cómo apoyados en la inmunofenotipificación tumoral, es posible profundizar el diagnóstico, emitir un pronóstico preciso y brindar al clínico tratante información que le ayude en sus decisiones terapéuticas. Palabras claves: linfoma canino, inmunofenotipo, diagnóstico, pronóstico.
Recibido: 13/05/2020

Aceptado: 16/10/2020 (cc) BY-NC

\section{Abstract}

Canine lymphoma is a malignant tumor that represents 7 to $24 \%$ of all malignancies and $80 \%$ of hematopoietic tumors. In humans and canines, $80-85 \%$ of lymphomas originate from neoplastic B-lymphocytes (B-cell lymphoma) and 15 to $20 \%$ are lymphomas generated from $\mathrm{T}$ lymphocytes. The latter are generally more aggressive, have shorter survival time and may need differential therapy. For this reason, knowing the tumor immunophenotype (origin of the tumor) allows establishing a more accurate prognosis and treatment appropriate to the type of tumor. In this context, immunophenotyping by flow cytometry performed from a lymph node puncture is a tool of great diagnostic value for the veterinary clinic. The aim of this work was to present two clinical cases of canines with a clinical and cytological diagnosis of lymphoma, which underwent flow cytometry to determine the cellular origin of the neoplasm, as well as complementary studies to determine local and systemic repercussions of the tumor process. The result of the immunophenotyping evidenced the presence of a CD4 + T-cell lymphoma in one of the patients and B-cell lymphoma in the other. At the systemic level, the patient with T-cell lymphoma presented neutrophilic leukocytosis and hypercalcemia, while in both patients increases of lactate dehydrogenase enzyme levels were observed. Based on these results, it is discussed how through the tumor immunophenotyping, it is possible to deepen in the diagnosis, to give an accurate prognosis and provide to the treating clinician a value information helping them in their therapeutic decisions.

Keywords: canine lymphoma, immunophenotype, diagnosis, prognosis. 


\section{Introducción}

El linfoma es un tumor maligno de origen linfoide que en caninos representa del 7 al 24\% del total de neoplasias (Withrow et al., 2013) y el $80 \%$ de los tumores hematopoyéticos (Aniolek et al., 2014; Meza y García, 2014). Su origen se considera multifactorial, y su aparición se ha asociado tanto a factores ambientales como genéticos (Meza y García, 2014; Modiano et al., 2005). Si bien se ha reportado que algunos caninos con linfoma de células $\mathrm{B}$ presentaron coinfección con gammaherpesvirus (Huang et al., 2012); hasta la fecha, no se ha determinado la relevancia de los agentes virales en la patogénesis del mismo (Waugh et al., 2015).

La incidencia del linfoma ha aumentado en las últimas décadas, alcanzando hasta 125 casos cada 100.000 de perros por año en Reino Unido (Dobson et al., 2002). Se presenta con más frecuencia en perros de 6 a 12 años de edad y el riesgo es mayor en las razas Bóxer, Doberman, Rottweiler y Boyero de Berna (Comazzi et al., 2018). La presentación multicéntrica es la más frecuente en perros (más del 80\% de los casos), y se caracteriza por presentar linfadenopatía generalizada (linfonódulos superficiales y profundos), la cual puede eventualmente afectar el parénquima hepático, el bazo y/o la médula ósea (Marconato et al., 2012). Los linfonódulos pueden aumentar hasta 10 veces su tamaño y en general son indoloros y móviles. La linfadenopatía puede estar acompañada de pérdida de peso, anorexia, letargo, fiebre y/o síntomas asociados a cuadros obstructivos u oclusivos generados por el crecimiento tumoral (Zandvliet, 2016).

Histológicamente, el linfoma es una neoplasia compleja cuyo origen puede estar asociado a distintos tipos y/o subtipos celulares vinculados con el desarrollo de los linfocitos B y T. En humanos y caninos, la incidencia de linfomas B (80-85\%) es ampliamente superior a los de estirpe T (15-20\%), reportándose diferente sensibilidad al tratamiento, calidad de vida y pronóstico entre ambos fenotipos (Seelig et al., 2016; Valli et al., 2011). Algunos autores sugieren una asociación entre el fenotipo tumoral, la sobrevida y el área geográfica donde habitan los caninos afectados (Wilson-Robles et al., 2017). Además, pareciera existir una clara diferencia racial (influenciada por rasgos heredables), en cuanto al fenotipo tumoral (Bennett et al., 2018; Ito et al., 2014), presentando las razas asiáticas linfomas de estirpe $T$ casi exclusivamente, mientras que las razas europeas desarrollan principalmente linfomas de células B (Modiano et al., 2006).

El diagnóstico clínico de linfoma se confirma por citología y/o histopatología. En el perro, la citología por punción con aguja fina (PAF) es la técnica de elección, por ser mínimamente invasiva y confirmar el $90 \%$ de los linfomas de alto grado (Marconato et al., 2012). No obstante, es necesaria la inmunofenotipificación celular de ese linfoma para definir el tratamiento y emitir un pronóstico. Esta puede realizarse por citometría de flujo (a partir de las muestras obtenidas por PAF) o por inmunohistoquímica. Si bien la inmunohistoquímica es económica, requiere sedación o anestesia del paciente para la obtención de la muestra, procedimiento poco aceptado por sus propietarios. Por otra parte, la citometría de flujo permite una rápida evaluación cuantitativa y cualitativa de la población de células hematopoyéticas, evaluando el tamaño celular, la expresión simultánea de diferentes antígenos en la misma población, así como también la evaluación cuantitativa de cada uno de ellos (Comazzi y Gelain., 2011; Gelain et al., 2008; Martini et al., 2013). La identificación de antígenos de superficie en los linfocitos neoplásicos denominados clusters de diferenciación (CD), permiten su tipificación y determinar el grado de indiferenciación. Algunos $\mathrm{CD}$, están presentes en todas las células linfáticas; mientras que otros se expresan exclusivamente en subpoblaciones de linfocitos T o B (Aniolek et al., 2014; Aresu et al., 2013; Martini et al., 2013).

Se ha reportado que la expresión de estos CD está asociada a la agresividad tumoral (Avery et al., 2014) y a la respuesta al tratamiento (Teske et al., 1994; Valli et al., 2013). Es así que el protocolo CHOP (ciclofosfamida, vincristina, doxorrubicina, prednisona), usado de rutina para tratar linfomas $\mathrm{B}$, tiene menor éxito en linfomas T de alto grado (Aresu et al., 2013; Beaver et al., 2010; Marconato, 2011). Asimismo, está demostrado que los linfomas agresivos de células $\mathrm{T}$ tienen peor pronóstico que aquellos de células $\mathrm{B}$, debido a que presentan características clínicas adversas al momento del diagnóstico y una mayor incidencia de recaída (Frantz et al., 2013; Milanovic et al., 2012; Teske et al., 1994). En este mismo sentido, Valli et al. (2013) reportaron que de 992 perros con linfoma, los pacientes con linfoma $\mathrm{T}$ agresivo tuvieron una supervivencia media inferior que los linfomas B agresivos (162 y 215 días, respectivamente). Resultados similares fueron encontrados en un estudio previo con 49 perros con linfoma donde los pacientes con fenotipo $\mathrm{T}$ tuvieron una supervivencia 4 veces inferior al fenotipo B (Dobson et al., 2001).

El objetivo de este trabajo es mostrar como la inmunofenotipificación del linfoma en caninos a través de la citometría de flujo, permite dirigir el tratamiento según la estirpe celular que da origen al tumor y emitir un pronóstico certero. Para dar cumplimiento a nuestro objetivo, presentaremos dos casos clínicos de linfoma en caninos con diferente inmunofenotipo, atendidos en el Hospital Escuela de la Facultad de Veterinaria, UdelaR.

\section{Materiales y métodos}

\section{Casos clínicos}

Se presentan dos casos clínicos de hembras caninas con linfoma que llegaron a consulta al Hospital de la Facultad de Veterinaria (UdelaR) derivados de clínicas particulares con diagnóstico clínico de linfoma. Cabe señalar que se cuenta con consentimiento escrito por parte de los propietarios de los caninos para publicar datos de los mismos. Uno de los casos correspondió a una perra cruza, de 12 años de edad, $11 \mathrm{Kg}$ de peso, de nombre "Lila"; y el otro caso a una Golden Retriever, de 5 años de edad, 33 $\mathrm{Kg}$ de peso, llamada "Catalina" (Figura 1). Al examen clínico realizado en ambas perras se evidenció que todos los nódulos 
linfáticos periféricos estaban aumentados de tamaño, presentaban movilidad y no mostraban dolor a la palpación. En el caso de Catalina, la linfadenopatía fue menos marcada y se acompañó de una clara depresión del sensorio. No se observaron particularidades en la coloración de las mucosas, temperatura corporal, así como tampoco en la frecuencia cardíaca y respiratoria en ninguna de las perras.

Las pacientes no recibieron tratamiento quimioterápico previo a la consulta en la Facultad de Veterinaria.
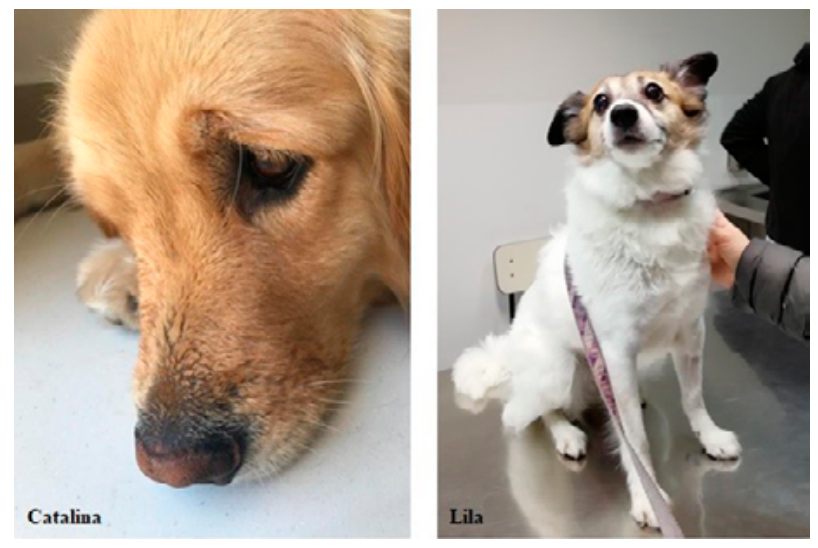

Figura 1: Imágenes de las pacientes al día de la consulta.

"Catalina", canino, Golden Retriever, hembra, 5 años, 33

Kg. "Lila”, canino, cruza, hembra, 12 años, $11 \mathrm{Kg}$.

\section{Toma de muestras}

Se extrajeron $5 \mathrm{~mL}$ de sangre de la vena cefálica a cada paciente, con agujas $21 \mathrm{G}$ y se dividieron en 2 submuestras. Una de ellas se colocó en un tubo con EDTA para la realización de un hemograma completo, y la otra en un tubo sin anticoagulante para la determinación de la calcemia, actividad sérica de la enzima lactato deshidrogenasa (LDH) y la realización del proteinograma. Se realizaron dos biopsias de linfonódulo a cada una de las pacientes, una de las cuales se utilizó para confirmar el diagnóstico por citología y la otra para realizar el inmunofenotipo. Las punciones se obtuvieron por PAF de linfonódulos poplíteos utilizando una jeringa de $5 \mathrm{~mL}$ y una aguja hipodérmica de $21 \mathrm{G}$. Para el estudio del inmunofenotipo el contenido extraído se colocó en un tubo eppendorf con EDTA y $1 \mathrm{~mL}$ de PBS (buffer fosfato salino, $\mathrm{pH}$ 7.4).

\section{Estudios paraclínicos}

El hemograma se realizó en un contador hematológico (Orphée, Mythic 18 Vet, Ginebra, Suiza). Para el recuento diferencial de los leucocitos y valoración de la morfología celular se realizó un frotis sanguíneo teñido con May Grunwald - Giemsa.

La calcemia y la actividad de la enzima LDH se determinaron en un equipo semiautomático, CB 350i (Wiener lab Group, Rosario, Argentina, números de referencia 12570 y 23580, respectivamente). En una submuestra de suero se realizó el proteinograma electroforético capilar en un equipo automático (Minicap, Sebia, Barcelona, España, número de referencia 2203).

\section{Imagenología}

Ambas pacientes por indicación del clínico veterinario particular actuante concurrieron a consulta con ecografía de abdomen y radiografía de tórax.

\section{Inmunofenotipo por citometría de flujo}

A partir de poblaciones de linfocitos provenientes de punciones de nódulos linfáticos se configuró la estrategia de "gate", basada en el tamaño y la granularidad de los mismos en un gráfico de puntos (dot plot) FSC (dispersión frontal) vs SSC (dispersión lateral). Se estableció un umbral de residuos (debris) para eliminar los eventos que tienen propiedades de dispersión frontal y lateral bajas (threshold 600.000 en FSC-H). La adquisición de las muestras se realizó en modo "slow" para garantizar que las células pasen de a una frente al haz de láser y sean debidamente excitadas. Además, se configuró un gráfico para eliminar dupletes (FSC-H vs FSC-A). Cuando existió superposición espectral entre fluorocromos se realizó un proceso de compensación del equipo a través del método de igualación de medianas (factor de compensación: FL2 vs FL1 9.1 \% y FL1 vs FL2 4.75\%). Para definir el punto de corte en la intensidad de fluorescencia de la población positiva y la negativa se utilizó el control FMO (fluoresence minus one). La intensidad de fluorescencia fue evaluada como la fluorescencia media en el citograma FSC $v s$ el canal de fluorescencia correspondiente (FL-1, FL-2, FL-3 o FL-4) y se expresaron como positivos cuando al menos el $85 \%$ de los eventos evaluados mostraron una fluorescencia mayor al control, como ya fue descripto por Gelain et al. (2008). Para evitar la muerte celular o una menor intensidad de expresión de algunos marcadores de superficie, las muestras para citometría fueron procesadas dentro de las 12 horas de extraídas (Jalla et al., 2004).

Una vez recibidas las muestras, se evaluó la calidad de las mismas en cuanto a cantidad de células obtenidas y viabilidad utilizando el citómetro de flujo Accuri C6 (BD BIOSCIENCES California, EEUU). Las muestras obtenidas de ambas pacientes fueron satisfactorias, presentando entre 500.000 a 1.000000 de células totales y una viabilidad superior al $80 \%$ determinada con tinción con Ioduro de propidio $(10 \mu \mathrm{g} / \mathrm{mL}$ - Invitrogen, by Thermo Fisher Scientific, Massachusetts, EEUU, número de catálogo P1304MP).

Con el fin de realizar la fenotipificación de las muestras provenientes de Lila y Catalina, se adquirieron un mínimo de 5000 linfocitos y se utilizaron anticuerpos monoclonales primarios específicos para la especie canina (BIO-RAD, USA) dirigidos contra antígenos de diferenciación celular CD34, CD45, CD3 y CD5, CD4, CD8, CD21 (Tabla 1). La incubación de todos los anticuerpos se realizó siguiendo las recomendaciones del fabricante (BIO-RAD), utilizando $5 \mu \mathrm{L}$ de cada anticuerpo en $50 \mu \mathrm{L}$ de muestra, en un ambiente oscuro durante 45 minutos y a temperatura ambiente. A continuación, se realizó la lisis de glóbulos rojos, utilizando $1 \mathrm{~mL}$ de buffer de lisis (Quicklysis, Cytognos, VISUR, Salamanca, España, catálogo CYT-QL-1), en oscuridad durante 10 minutos. Una vez completada la lisis, 
las células nucleadas estuvieron prontas para ser adquiridas en el citómetro de flujo. Las muestras se analizaron siempre en duplicado. Como control negativo se incluyó un tubo con el mismo tipo de muestra y tratado bajo las mismas condiciones, pero al que no se le añadió anticuerpos.

\section{Resultados}

El examen citológico de ambas pacientes confirmó el diagnóstico clínico presuntivo de linfoma. La evaluación citológica de ambas pacientes mostró una población celular compuesta mayoritariamente por linfoblastos, y la presencia de moderada cantidad de cuerpos linfoglandulares (restos citoplasmáticos) (Figura 2).

El hemograma de "Lila" no presentó anormalidades, mientras que el de "Catalina" evidenció leucocitosis neutrofílica (Tabla 2).

La bioquímica sérica mostró en ambos casos actividad aumenta-

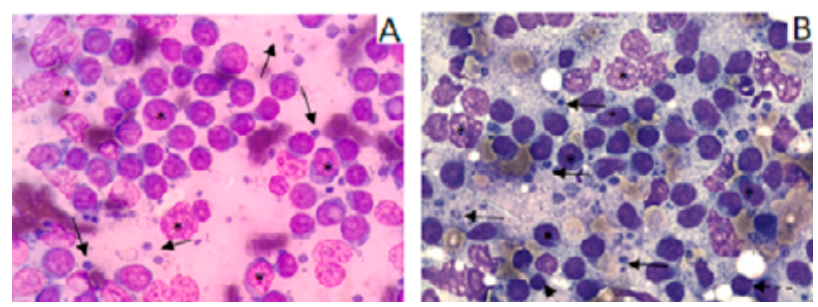

Figura 2: Citología de linfonódulo poplíteo obtenida por PAF y teñida con May-Grunwald Giemsa. (A, "Catalina";

B, "Lila”). En ambas citologías, la población celular está mayoritariamente compuesta por linfoblastos $(*)$ y se observan cuerpos linfoglandulares de tamaños variables (flecha sólida) y linfocitos normales (flecha punteada). da de la enzima LDH y a su vez "Catalina" presentó una marcada hipercalcemia (Tabla 2). El proteinograma electroforético capilar (PEF), descartó la presencia de gammapatías en ambas pacientes.

El estudio ecográfico realizado en "Lila" mostró que ambos riñones presentaban tamaño y ecotextura conservada y cambios acordes a la edad. El bazo se mostró levemente aumentado de

Tabla 2: Análisis hematológicos y bioquímica sérica de las pacientes con linfoma

\begin{tabular}{||lcl|}
\hline Parámetros & Lila Catalina & $\begin{array}{l}\text { Rango de Referencia } \\
\text { (Oregon State Uni- } \\
\text { versity) }\end{array}$ \\
\hline
\end{tabular}

\begin{tabular}{|c|c|c|c|}
\hline Hematocrito (\%) & 49,5 & 38,3 & $37-55$ \\
\hline Hemoglobina (g/dL) & 17,7 & 17 & $12-18$ \\
\hline Recuento Eritrocitos $\left(10^{6} / \mu \mathrm{L}\right)$ & 6,52 & 6,01 & $5,5-8,5$ \\
\hline VCM (fL) & 75,9 & 63,7 & $60-77$ \\
\hline HCM (pg) & 27,1 & 28,3 & $19,5-24,5$ \\
\hline CHCM $(\mathrm{g} / \mathrm{dL})$ & 35,8 & 44,4 & $32-36$ \\
\hline Plaquetas $\left(* 10^{5} / \mu \mathrm{L}\right)$ & 231 & 232 & $200-900$ \\
\hline Recuento leucocitos $(/ \mu \mathrm{L})$ & 7700 & 20400 & $6000-17000$ \\
\hline Neutrófilos $(/ \mu \mathrm{L})$ & 5852 & 17544 & $3000-11400$ \\
\hline Linfocitos $(/ \mu \mathrm{L})$ & 1309 & 1632 & $1000-4800$ \\
\hline Monocitos $(/ \mu \mathrm{L})$ & 385 & 1020 & $150-1350$ \\
\hline Eosinófilos $(/ \mu \mathrm{L})$ & 154 & 204 & $100-750$ \\
\hline Basófilos $(/ \mu \mathrm{L})$ & 0 & 0 & Raros \\
\hline Calcio (mg/dL) & 9,95 & 14,82 & $8-11,4$ \\
\hline LDH (UI/L) & 614 & 268 & $30-236$ \\
\hline
\end{tabular}

Tabla 1: Marcadores de leucocitos utilizados para detectar linfoma canino por citometría de flujo

\begin{tabular}{|c|c|c|c|c|c|}
\hline Anticuerpo & Fluorocromo & Tipo celular & Proveedor & Código & Referencias \\
\hline $\begin{array}{l}\text { CD3 } \\
\text { Mouse anti dog }\end{array}$ & FITC & Linfocitos $\mathrm{T}$ & BIORAD & MCA1774F & $\begin{array}{c}\text { Papakonstantinou e } \\
\text { al., } 2013\end{array}$ \\
\hline $\begin{array}{l}\text { CD4 } \\
\text { Rat anti dog }\end{array}$ & FITC & $\begin{array}{l}\text { Linfocitos T colaboradores Neutrófilos, } \\
\text { monocitos y células dendríticas caninas }\end{array}$ & BIORAD & MCA1038F & Martini et al., 2013 \\
\hline $\begin{array}{l}\text { CD5 } \\
\text { Rat anti dog }\end{array}$ & FITC & $\begin{array}{l}\text { Linfocitos T. En algunas especies, CD5 } \\
\text { puede estar presente en linfocitos B }\end{array}$ & BIORAD & MCA1037F & Aniolek et al., 2014 \\
\hline $\begin{array}{l}\text { CD8 } \\
\text { Rat anti dog }\end{array}$ & PE & $\begin{array}{c}\text { Linfocitos T citotóxicos. También en el } \\
\text { subgrupo NK }\end{array}$ & BIORAD & MCA1039PE & Martini et al., 2013 \\
\hline $\begin{array}{l}\text { CD21 } \\
\text { Mouse anti dog }\end{array}$ & PE & $\begin{array}{l}\text { Linfocitos B maduros. Ausente en célu- } \\
\text { las plasmáticas }\end{array}$ & BIORAD & MCA1781PE & $\begin{array}{c}\text { Comazzi y Gelain., } \\
2011\end{array}$ \\
\hline $\begin{array}{l}\text { CD34 } \\
\text { Mouse anti dog }\end{array}$ & PE & Células madres hematopoyéticas & BIORAD & MCA2411PE & $\begin{array}{c}\text { Comazzi y Gelain, } \\
2011\end{array}$ \\
\hline $\begin{array}{l}\text { CD45 } \\
\text { Rat anti dog }\end{array}$ & $\mathrm{APC}$ & $\begin{array}{l}\text { Todas las células madres hematopoyéti- } \\
\text { cas (excepto eritroides) }\end{array}$ & BIORAD & MCA1042APC & Aniolek et al., 2014 \\
\hline
\end{tabular}


tamaño, con bordes lisos y parénquima heterogéneo. Se halló un nódulo esplénico hipoecocico de bordes mal definidos (5.2 $\mathrm{mm}$ ), de aspecto hiperplásico/neoplásico. El hígado y la vesícula biliar no mostraron alteraciones evidentes. Los linfonódulos ilíacos, yeyunales y gástrico se encontraron aumentados de tamaño, con forma redondeada y aspecto reactivo/infiltrativo. La ecografía de la otra paciente no presentó particularidades. En las radiografías, solo se observó en "Catalina" un desplazamiento dorsal de la tráquea en región craneal sugestivo de adenomegalia mediastínica moderada.

La inmunofenotipificación por citometría de flujo en el caso de "Catalina" resultó compatible con linfoma originado en linfocitos $\mathrm{T}$ helpers, caracterizado por marcación positiva a antígenos CD45, CD3, CD5 y CD4 y negativa a CD8 (T citotóxicos) y CD21 (específico de linfocitos B) (Figura 3A). Distinto fue lo observado en la paciente "Lila" donde el estudio por citometría concluyó que se trataba de un linfoma originado en linfocitos $\mathrm{B}$, presentando marcación positiva solamente para los antígenos CD21 y CD45 (Figura 3B).

El tratamiento de Lila (linfoma B) comenzó con 11 semanas de protocolo COP (ciclofosfamida $200 \mathrm{mg} / \mathrm{m}^{2} / \mathrm{IV} /$ cada 21 días + vincristina $0.7 \mathrm{mg} / \mathrm{m}^{2} / \mathrm{IV} /$ cada 7 días + prednisona $1 \mathrm{mg} / \mathrm{kg} / \mathrm{día} /$ PO), hasta que tuvo una recaída y los linfonódulos comenzaron a aumentar de tamaño (pérdida de remisión). Al mismo tiempo se relacionó la ciclofosfamida con aparición de cistitis hemorrágica, efecto adverso ya descrito para este fármaco (Haldar et al., 2014) y fue sustituida por clorambucilo $\left(20 \mathrm{mg} / \mathrm{m}^{2} / \mathrm{PO}\right)$ en el protocolo terapéutico. Luego de recibir dos ciclos de clorambucilo y vincristina y debido a que los linfonódulos continuaron aumentando de tamaño, comenzó a administrarse doxorrubicina ( $1 \mathrm{mg} / \mathrm{Kg} / \mathrm{IV})$. Una semana después los linfonódulos seguían aumentando de tamaño por lo que se administró lomustina y luego de una semana se volvió a administrar vincristina.

El tratamiento de Catalina (linfoma T) comenzó con la administración de L-Asparginasa (una sola dosis de $400 \mathrm{UI} / \mathrm{kg} / \mathrm{SC}$ ), acompañada de prednisona (40mg/día/PO) y omeprazol (20mg/ día/PO). A la semana siguiente se administró Vincristina (1 dosis de $0.7 \mathrm{mg} / \mathrm{m}^{2} / \mathrm{IV}$ ) y luego del mismo período recibió una dosis de lomustina ( $75 \mathrm{mg} / \mathrm{m}^{2} / \mathrm{PO}$ ). En ese momento se disminuyó la dosis de prednisona a $30 \mathrm{mg}$ por día, vía oral.

Finalmente luego de 233 días de sobrevida, se realizó la eutanasia de Lila debido a la pérdida de remisión y falta de respuesta a los tratamientos de segunda línea instaurados. Catalina falleció luego de 31 días de sobrevida debido a una descompensación sistémica.

\section{Discusión}

El diagnóstico definitivo del linfoma requiere una aproximación multifocal, involucrando aspectos clínicos, citológicos, histológicos y moleculares. El perfil hematológico así como la bioquímica sérica pueden mostrar una amplia gama de alteraciones aunque ninguna de ellas es patognomónica de la enfermedad (Gavazza et al., 2008). Los hemogramas realizados a las pacientes no mostraron alteraciones en la serie roja, sin embargo una de ellas presentaba una leucocitosis neutrofílica. Este hallazgo es una alteración hematológica frecuente en pacientes con linfoma cuando existe una condición inflamatoria con participación neoplásica de varios tejidos (Gavazza et al., 2008).

La hipercalcemia en perros con linfoma se presenta en el 20 a $40 \%$ de los casos y casi exclusivamente en linfomas de estirpe T (Aresu et al., 2013; Zandvliet, 2016). En concordancia con lo reportado, la paciente con linfoma $\mathrm{T}$ fue la que presentó valores
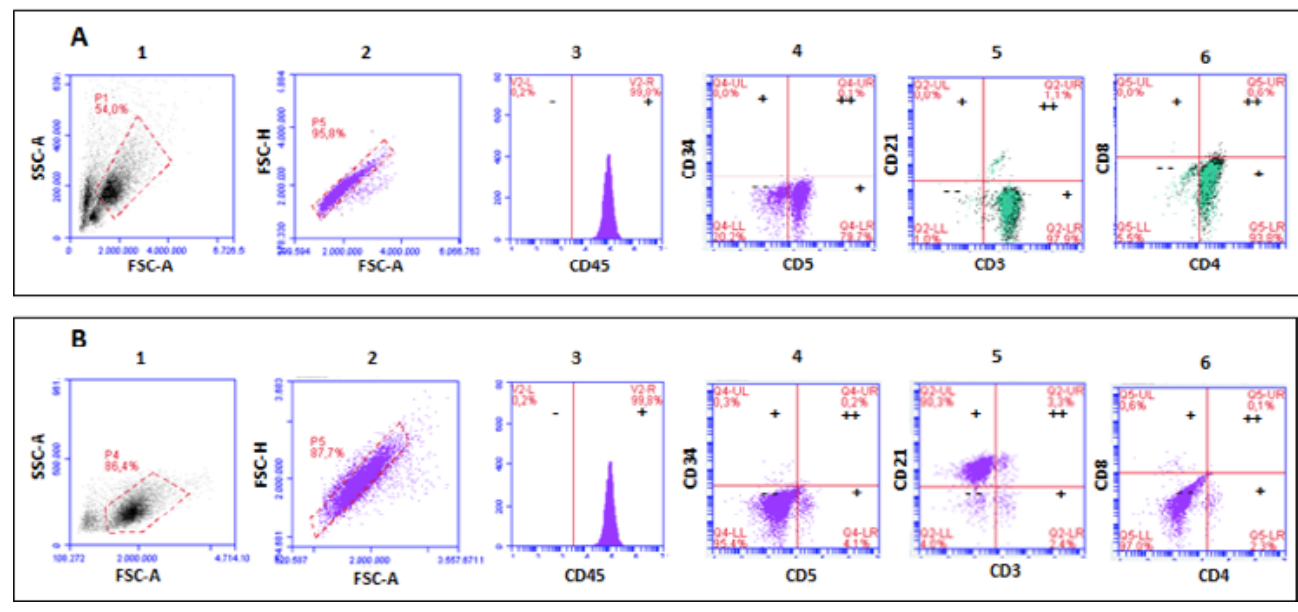

Figura 3: Representación gráfica de las citometrías de flujo realizadas en punciones de linfonódulos de ambas pacientes (paneles A у B). Los eventos se muestran en función de las propiedades morfológicas (SSC vs FSC) (A.1 y B.1), y se establece un gate para eliminar debris y plaquetas (P1 y P4). Se establece un gráfico (FSC-A vs FSC-H) para eliminar dupletes (P5) en la población de células contenidas en el gate anterior (A.2 y B.2). Los histogramas (A.3 y B.3) muestran que la población linfocitaria estudiada en ambos casos fue positiva a CD45. Panel A: Paciente "Catalina" diagnóstico de Linfoma T CD4+; muestra positiva a los antígenos CD5 (A.4), CD3 (A.5), CD4 (A.6) y negativa a CD8 (A.6) y CD21 (A.5). Panel B: Paciente "Lila" con linfoma B; muestra positiva a CD21 (B.5) y negativa a CD5 (B.4), CD3 (B.5) y CD4, CD8 (B.6). 
elevados de calcio en sangre. Goldner (2016) explica este hecho por la síntesis de PTHrP (péptido relacionado a la parathormona) a cargo de los linfocitos $\mathrm{T}$ neoplásicos, que estimula la resorción ósea. Para algunos autores dicho aumento puede asociarse a un peor pronóstico (Marconato et al., 2011), mientras que otros consideran que la hipercalcemia en los linfomas $\mathrm{T}$ no tiene efecto negativo adicional en la respuesta al tratamiento o la supervivencia (Rebhun et al., 2011).

El aumento de la actividad sérica de la LDH encontrado en nuestras pacientes también es un hallazgo frecuente en perros con linfoma (Marconato et al., 2009; Zanatta et al., 2003). En estos pacientes ocurre un "switch" hacia el metabolismo anaerobio (glucólisis anaerobia), actuando esta enzima en la conversión del lactato en piruvato, mecanismo necesario para poder sostener el rápido crecimiento tumoral (Fantin et al., 2006; Marconato et al., 2009).

Los anticuerpos más comúnmente utilizados para estudiar el linaje celular de los linfomas en caninos incluyen CD45 (panleucocitario), CD20, CD21, CD79a para células B y CD3, CD5, CD4 y CD8 para las células T (Aniolek et al., 2014; Aresu et al., 2013; Avery et al., 2014; Comazzi y Gelain, 2011; Martini et al., 2013). La selección de anticuerpos utilizados en el presente trabajo comprendió la mayoría de los marcadores celulares disponibles en el mercado y recomendados por la literatura internacional. Papakonstantinou et al. (2013) reportaron que el uso de un solo marcador para cada tipo celular (CD3 y CD21) es suficiente para inmunofenotipificar al $92 \%$ de los linfomas caninos. Sin embargo, la utilización de un panel amplio de anticuerpos permite resultados más concluyentes y la detección de aberraciones de interés pronóstico. Por ejemplo, existe una variante en los linfomas T que no expresan CD45 (linfomas de zona $\mathrm{T}$ indolentes), que se caracterizan por presentar un buen pronóstico incluso sin tratamiento (Avery et al., 2014; Martini et al., 2013; Seelig et al., 2014). El panel de anticuerpos empleados en nuestro servicio de inmunofenotipificación de la Facultad de Veterinaria (UdelaR), ha permitido identificar por primera vez a nivel nacional algunas de estas variantes de linfoma brindando herramientas para ajustar el tratamiento y el pronóstico en función del resultado diagnóstico.

El tratamiento de linfoma tiene por finalidad dar al paciente una mejor calidad de vida y buscar la remisión de la enfermedad por el mayor tiempo posible. En general los perros que no reciben tratamiento, no sobreviven más de 4 a 6 semanas luego de realizado el diagnóstico (Withrow y Macewen, 2001). Con un tratamiento adecuado y dependiendo del tipo de tumor se puede esperar una sobrevida mayor, aunque en la mayoría de los casos no supera los 12 meses post diagnóstico (Vail y Young, 2007; Young y Rickinson, 2004).

En el presente trabajo, la inmunofenotipificación permitió en la paciente con linfoma T, implementar un tratamiento con L-Asparginasa y Lomustina, fármacos no dependientes de la proteína MDR-1 (multidrug resistance protein1) (Morgan et al., 2018; Rassnick et al., 2002). Esta proteína es altamente expresada por los linfocitos T neoplásicos y es capaz de disminuir la concentración intracelular de la mayoría de los fármacos utilizados en los protocolos convencionales (Klimecki et al., 1994). La paciente con linfoma $\mathrm{B}$, fue tratada con ciclofosfamida, vincristina y prednisona (protocolo COP), ampliamente aceptado para este fenotipo tumoral.

El pronóstico de la enfermedad está condicionado por el inmunofenotipo tumoral, presentando en general, los linfomas $\mathrm{T}$ de alto grado peor pronóstico (Frantz et al., 2013; Milanovic et al., 2012; Teske et al., 1994). En este trabajo, observamos una marcada diferencia en los tiempos de sobrevida de las pacientes lo cual se explica por la estirpe celular diferente que dio origen al tumor (inmunofenotipo B vs T). En el caso de "Catalina" (estirpe T) fue inferior incluso a lo reportado para este fenotipo a nivel internacional (Avery et al., 2014; Valli et al., 2013). Cabe señalar que ésta paciente con linfoma $T$ presentaba además hipercalcemia y una masa en mediastino, estando ambos hallazgos asociados a mal pronóstico (Garret et al., 2002; Marconato et al., 2011; Vail et al., 2013). En el caso de la paciente "Lila" (linfoma B) la sobrevida alcanzada fue acorde a lo reportado para su fenotipo y al tratamiento instaurado (Vail y Young, 2007; Young y Rickinson, 2004).

\section{Conclusión}

El empleo del inmunofenotipo resulta un paso ineludible en la evaluación diagnóstica del linfoma canino, ya que complementa el estudio citológico realizado de rutina y permite implementar una estrategia terapéutica que contemple la estirpe celular que da origen a la neoplasia. Utilizar la citometría de flujo para este propósito tiene como ventaja adicional la rapidez con que se realiza el estudio y se emite el resultado, permitiendo instaurar el tratamiento en forma inmediata, aspecto relevante en este tipo de neoplasia donde la sobrevida en general es corta.

\section{Agradecimientos}

Agradecemos a la Dra. Ana Meikle por sus aportes y la corrección de este manuscrito.

\section{Referencias bibliográficas}

Aniolek, O., Gajewski, Z. y Ginzinski S. (2014). Application of flow cytometry in diagnosing lymphomas in dogs and cats. Central European Journal of Immunology, 39(3), 327-330.

Aresu. L., Martini, V., Rossi, F., Vignoli, M., Sampaolo M, Arico A...Marconato L. (2013). Canine indolent and aggressive lymphoma: clinical spectrum with histologic correlation. Veterinary and Comparative Oncology, 13(4), 348-62.

Avery, P.R., Burton, J., Bromberek, J.L., Seelig, D.M., Elmslie, R., Correa... Avery, A.C. (2014). Flow cytometric characterization and clinical outcome of CD4+ T-cell lymphoma in dogs: 67 Cases. Journal of Veterinary Internal Medicine, 28 


\section{(2), 538-546.}

Beaver, L.M., Strottner, G. y Klein, M.K. (2010). Response rate after administration of a single dose of doxorubicin in dogs with B-cell or T-cell lymphoma: 41 cases (2006-2008). Journal of the American Veterinary Medical Association, 237(9), 1052-1055.

Bennett, P.F., Taylor, R. y Williamson, P. (2018). Demographic risk factors for lymphoma in Australian dogs: 6201 cases. Journal of the American Veterinary Medical Association, 32(6), 2054-2060.

Comazzi, S. y Gelain, M.E. (2011). Use of flow cytometric immunophenotyping to refine the cytological diagnosis of canine lymphoma. Veterinary Journal, 188 (2), 149-155.

Comazzi, S., Marelli, S., Cozzi, M., Rizzi, R., Finotello, R, Henriques J...Teske, E. (2018). Breed-associated risks for developing canine lymphoma differ among countries: an European canine lymphoma network study. BMC Veterinary Research, 14 (1), 232.

Dobson, J.M., Blackwood, L.B., Mc Innes, D.E., Bosrock, E., Nichollst, P., Hoather, M. y Tom A.D. (2001). Prognostic variables in canine multicentric lymphosarcoma. Journal of Small Animal Practice, 42 (8), 377-384.

Dobson, J.M., Samuel, S., Milstein, H., Rogers, K. y Wood, J.L. (2002). Canine neoplasia in the UK: estimates of incidence rates from a population of insured dogs. Journal of Small Animal Practice, 43, 240-246.

Fantin, V.R., St-Pierre, J. y Leder, P. (2006). Attenuation of LDH A expression uncovers a link between glycolysis, mitochondrial physiology, and tumor maintenance. Cancer Cell, 9 (6), 425-434.

Frantz, A.M., Sarver, A.L., Ito, D., Phang, T.L., Karimpour-Fard, A., Scott, M.C. ... Modiano J.F. (2013). Molecular Profiling Reveals Prognostically Significant Subtypes of Canine Lymphomas. Veterinay Pathology, 50 (4), 693-703.

Garrett, L.D., Thamm, D.H., Chun, R., Dudley, R. y Vail, D.M. (2002). Evaluation of a 6-month chemotherapy protocol with no maintenance therapy for dogs with lymphoma. Journal of Veterinary Internal Medicine, 16 (6), 704-709.

Gavazza, A., Lubas, G., Valori, E. y Gugliucci B. (2008). Retrospective survey of malignant lymphoma cases in the dog: clinical, therapeutical and prognostic features. Veterinary Research Communications, 32, 291-293.

Gelain, M.E., Mazzilli, M., Riondato, F., Marconato, L. y Comazzi, S. (2008). Aberrant phenotypes and quantitative antigen expression in different subtypes of canine lymphoma by flow cytometry. Veterinary Immunology and Immunopathology, 121, 179-188.
Goldner W. (2016). Cancer-Related Hypercalcemia. Journal of Oncology Practice, 12, 426-435.

Haldar, S., Dru, C. y Bhowmick, N.A. (2014). Mechanisms of hemorrhagic cystitis. American Journal of Clinical and Experimental Urology, 2(3), 199-208.

Huang, S., Kozak, P.J., Kim, J., Habineza-Ndikuyeze, G., Meade, C., Guarnier-Hausser, A... Mason. (2012). Evidence of an oncogenic gammaherpesvirus in domestic dogs. Virology, 427 (2), 107-117.

Ito, D., Frantz, A.M. y Modiano, J.F. (2014). Canine lymphoma as a comparative model for human non-Hodgkin lymphoma: recent progress and applications. Veterinary Immunology and Immunopathology, 159(3-4), 192-201.

Jalla, S., Sazawal, S., Deb, S., Black, R.E., Das, S.N., Sarkar, A. y Bhan, M. (2004). Enumeration of lymphocyte subsets using flow cytometry: effect of storage before and after staining in a developing country setting. Indian Journal of Clinical Biochemistry, 19, 95-99.

Klimecki, W.T., Futscher, B.W., Grogan, T.M. y Dalton, W.S. (1994). P-glycoprotein expression and function in circulating blood cells from normal volunteers. Blood, 83(9), 2451-8.

Marconato, L., Crispino, G., Finotello, R., Mazzotti, S., Salerni, F. y Zini, E. (2009). Serum lactate dehydrogenase activity in canine malignancies. Veterinary and Comparative Oncology, 7 (4), 236-43.

Marconato, L. (2011). The staging and treatment of multicentric high-grade lymphoma in dogs: A review of recent developments and future prospects. Veterinary Journal, 188, 34-38.

Marconato, L., Stefanello, D., Valenti, P., Bonfanti, U., Comazzi, S., Roccabianca P...Zini, E. (2011). Predictors of long-term survival in dogs with high grade multicentric lymphoma. Journal of the American Veterinary Medical, 238, 480-485.

Marconato, L., Gelain, M.E. y Comazzi, S. (2012). The dog as a possible animal model for human non-Hodgkin lymphoma: a review. Hematological Oncology, 31(1), 1-9.

Martini, V., Poggi, A., Riondato, F., Gelain, M.E., Aresu, L. y Comazzi, S. (2013). Flow-cytometric detection of phenotypic aberrancies in canine small clear cell lymphoma. Veterinary and Comparative Oncology, 13(3), 281-287.

Meza, A.B. y García, L.E. (2014). Citología de linfonódulos. En Nuria de Buen de Arguero, Atlas de citopatología veterinaria (pp. 313-329). Buenos Aires: Intermédica.

Milanovic, N., Matkovic, S., Ristic, D., Jelic, S. y Petrovic, M. (2012). Significance of tumor burden, vascular endothelial growth factor, lactate dehydrogenase and beta- 2 microglobulin serum levels in advanced diffuse large B cell lymphoma. Journal BUON, 17 (3), 497-501. 
Modiano, J.F., Breen, M., Burnett, R.C, Parker, H.G., Inusah, S., Thomas, R...Avery A.C. (2005). Distinct B-cell and T-cell lymphoproliferative disease prevalence among dog breeds indicates heritable risk. Cancer Research, 65, 5654-5661.

Modiano, J.F., Breen, M., Avery, A.C. y London, C.A. (2006). Breed-specific canine lymphoproliferative diseases. En E.A. Ostrander, U. Giger y K. Lindblad-Toh (Eds.). The Dog and its Genome (pp. 439- 450). New York: Cold Spring Harbor Laboratory.

Morgan, E., O’Connell, K., Thomson, M. y Griffin, A. (2018). Canine $\mathrm{T}$ cell lymphoma treated with lomustine, vincristine, procarbazine, and prednisolone chemotherapy in 35 dogs. Veterinary and Comparative Oncology, 16(4), 622-629.

Papakonstantinou, S., Berzina, I., Lawlor, A., O’Neill, E.J. y O’Brien, P.J. (2013). Rapid, effective and user-friendly immunophenotyping of canine lymphoma using a personal flow cytometer. Irish Veterinary Journal, 66 (1), 6.

Rassnick, K.M., Mauldin, G.E., Al-Sarraf, R., Mauldin, G.N., Moore, A.S. y Mooney, S.C. (2002). MOPP chemotherapy for treatment of resistant lymphoma in dogs: a retrospective study of 117 cases (1989-2000). Journal of Veterinary Internal Medicine, 16 (5), 576-580.

Rebhun, R.B., Kent, M.S., Borrofka, S.A., Frazier, S., Skorupski, K. y Rodriguez, C.O. (2011). CHOP chemotherapy for the treatment of canine multicentric T-cell lymphoma. Veterinary and Comparative Oncology, 9, 38-44.

Seelig, D.M., Avery, P., Webb, T., Yoshimoto, J., Bromberek, J., Ehrhart, E.J. y Avery, A.C. (2014). Canine T-zone lymphoma: unique immunophenotypic features, outcome, and population characteristics. Journal of Veterinary Internal Medicine, 28, 878-886.

Seelig, D.M., Avery, A.N., Ehrhart, E.J. y Linden, M.A. (2016). The comparative diagnostic features of canine and human lymphoma. Veterinary Sciences, 3(2), 11.

Teske, E., de Vos, J.P., Egberink, H.F. y Vos, J.H. (1994). Clustering in canine malignant lymphoma. Veterinary Quarterly, 16, 134-136.

Vail, D. yYoung, K. (2007). Hematopoietic Tumors. En S. Wi-

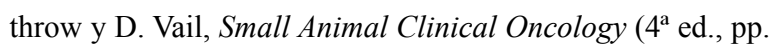
699-709). St. Louis: Elsevier.

Vail, D.M., Pinkerton, M.E. y Young, K.M. (2013). Hematopoietic tumors. En S.J. Withrow, D.M. Vail, R.L. Page (Eds.), Small Animal Clinical Oncology (5 $5^{\mathrm{a}}$ ed. pp. 608-688). St. Louis: Elsevier.

Valli, V.E., San Myint, M., Barthel, A., Bienzle, D., Caswell, J., Colbatzky, F...Vernau, W. (2011). Classification of canine malignant lymphomas according to the World Health Organi- zation criteria. Veterinay Pathololgy, 48, 198-211.

Valli, V.E., Kass, P.H., Myint, M.S. y Scott, F. (2013). Canine lymphomas: association of classification type, disease stage, tumor subtype, mitotic rate, and treatment with survival. Veterinary Pathology, 50(5), 738-748.

Waugh, E., Gallagher, A., McAulay, A.K., Henriques, J., Alves, M., Bell, A.J...Jarrett, R.F. (2015). Gammaherpesvirusses and canine lymphoma: no evidence for direct involvement in commonly occurring lymphomas. Journal of General Virology, 96, 1863-1872.

Wilson $\square$ Robles, H., Budke, C.M., Miller, T., Dervisis, N., Novosad, A., Wright, Z... Laver, T. (2017). Geographical differences in survival of dogs with non $\square$ Hodgkin lymphoma treated with a CHOP based chemotherapy protocol. Veterinary and Comparative Oncology, 15(4), 1564-1571.

Withrow, S.J y Macewen, E.G. (2001). Small Animal Clinical Oncology ( $3^{\mathrm{a}}$ ed.). Philadelphia: Saunders.

Withrow, S.J., Vail, D.M. y Page, R.L. (2013). Withrow \& MacEwen's Small Animal Clinical Oncology ( $5^{\mathrm{a}}$ ed.). St. Louis: Elsevier.

Young, L.S. y Rickinson, A.B. (2004). Epstein-Barr virus: 40 years on. Nature Reviews Cancer, 4 (10), 757-768.

Zanatta, R., Abatem O., D’Angelo, A., Miniscalco, B.y Mannelli, A. (2003). Diagnostic and prognostic value of serum lactate dehydrogenase (LDH) and $\mathrm{LDH}$ isoenzymes in canine lymphoma. Veterinary Research Communications, 27, 449-452.

Zandvliet, M. (2016). Canine lymphoma: a review, Veterinary Quarterly, 36 (2), 76-104.

\section{Notas de contribución:}

Rosina Sánchez es el autor principal, redactó el artículo, realizó la adquisición y análisis de datos y realizó la búsqueda bibliográfica. Martín Breijo y Paula Pessina colaboraron en igual medida en los procesos de diseño y revisión así como también en la corrección del manuscrito. Cecilia Amaral proporcionó los casos clínicos y colaboró en la corrección del texto.

El editor Cecilia Cajarville aprobó este artículo 